\title{
Vehicle-induced loads on traffic sign panels
}

\author{
A. Sanz-Andrés \\ J. Santiago-Prowald \\ C. Baker \\ A. Quinn
}

\begin{abstract}
The determination of the loads on traffic sign panels in the current standards does not, in general, take into account the vehicle-induced loads, as explained by Quinn, Baker and Wright (QBW in what follows) (J. Wind Eng. Ind. Aerodyn. 89 (2001) 831). On the other hand, a report from Cali and Covert (CC) (J. Wind Eng. Ind. Aerodyn. 84 (2000) 87) indicates that in highway sign support structures, vehicle-induced loads have led to premature failures in some cases. The aim of this paper is to present a mathematical model for the vehicle-induced load on a flat sign panel, simple enough to give analytical results, but able to explain the main characteristics of the phenomenon. The results of the theoretical model help to explain the behaviour observed in the experiments performed in previous studies.
\end{abstract}

\section{Introduction}

Full-scale measurements have been performed by Quinn, Baker and Wright (QBW) [1] in order to gather data on the vehicle-induced loading on traffic signs and pedestrian barriers. These data show that the wind-induced forces can be treated separately from the vehicle-induced loads (at least to first order) because the latter are generated largely by the transient pressure field induced by the vehicle motion relative to the sign and not by the motion of the air. The vehicle slipstreams tend to be weak at typical distances of road signs from the traffic stream. 


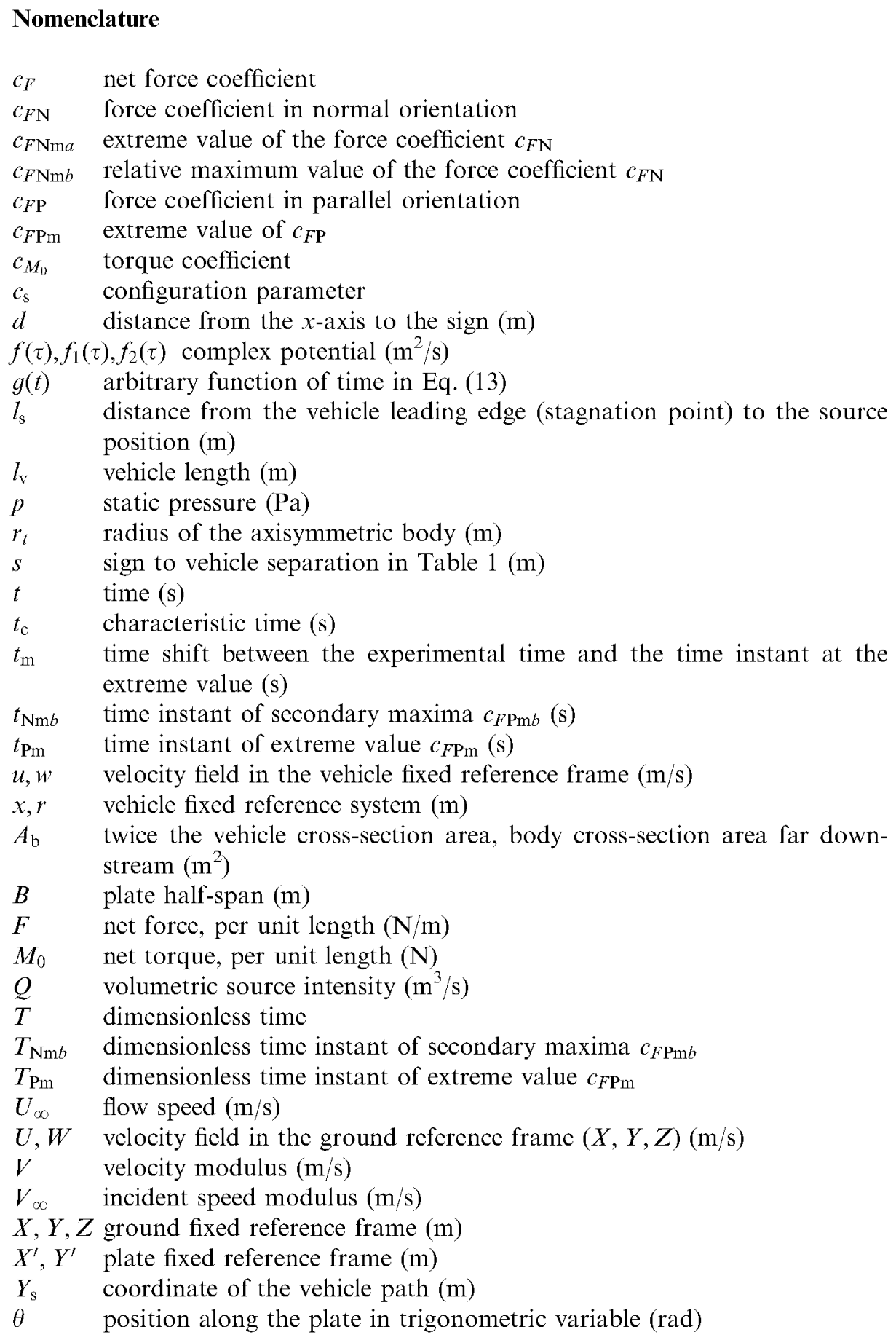




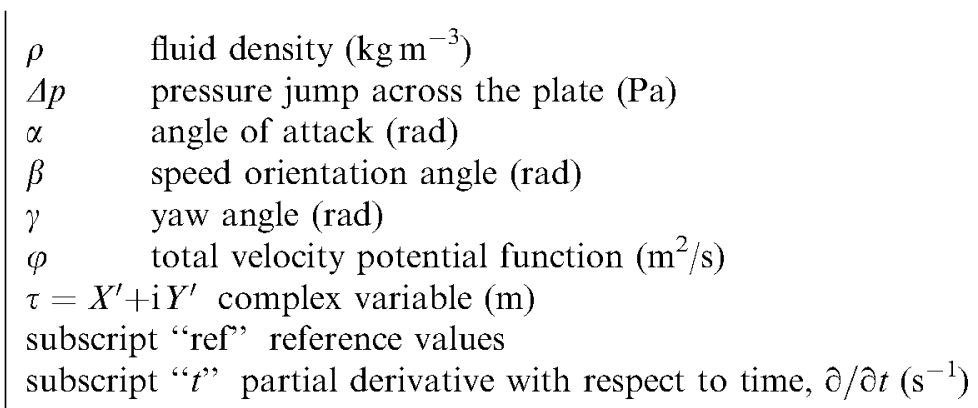

In the above-mentioned study, it is also concluded that the traffic sign shape does not have a significant influence on the vehicle-induced overall force coefficient value (when the force coefficient is referenced to the vehicle speed and the sign area) but the sign size does have an influence on the force coefficient (presumably because of different ground proximities). Another parameter that has an influence is the orientation of the sign with regard to the vehicle motion direction; signs placed normal to the road direction experience a force peak in the opposite direction to the vehicle motion, which occurs close to the vehicle leading edge passing the sign, whilst signs placed parallel to the road direction show a pushing out of the road when the vehicle is approaching $(t<0)$ and a pulling towards the road when the leading edge of the vehicle passes the plate.

Cali and Covert (CC) [2] report presents results of tests performed on sign and vehicle models (at 1:30 scale) for the horizontal loading produced by vehicle-induced gusts on overhead highway sign structures, with the vehicle model passing underneath. Several values of test parameters, such as the vehicle speed and shape, vehicle length, $l_{v}$, and height of the sign were considered. In these tests a set of characteristics of the loads were identified, which seems to be universal for all kind of vehicles, e.g. the maximum load appears in the opposite direction to the vehicle motion and occurs when the leading edge passes close to the front of the sign, and afterwards the load changes in sign, in a similar way as described by QBW.

While there is a small number of experimental datasets (QBW, CC), there is a lack of theoretical models that can help understand the underlying phenomena. In this paper a simple theoretical model is presented, with the aim to explain the phenomena observed in the performed experiments. Although the developed model is simple, the theoretical results allow the predictions (in some cases) of values of the parameters involved that are close to the experimental observations. This fact suggests the possibility of future development of more detailed models of both the vehicle and sign geometry.

The model developed here follows the method employed in Ref. [3] to study the train-induced pressure on pedestrians. In order to develop the theoretical model, the following assumptions have been considered:

(1) The flow generated by the vehicle motion is a potential flow. Emphasis is placed on the nose effect, thus the effect of the wake, being outside this restriction, can be safely neglected. 
(2) The three-dimensional potential flow generated by the vehicle motion is represented as that produced by a moving source, with intensity $Q$, which is determined by considering the vehicle cross-section area and the vehicle speed. The axisymmetric flow thus obtained represents the vehicle itself, as the upper half of a horizontal axisymmetric body. The horizontal meridian plane represents the ground. This model is crude but allows obtaining analytical solutions where the influence of the involved parameters can be easily described.

(3) The size of the traffic sign (a plate) is much smaller than the vehicle cross-section area, and is far enough from the source so that the influence of the sign on the flow generated by the source can be neglected. Based on this assumption the complete problem can be split into two simplified problems: (1) a uniform, steady, incident flow around the vehicle (without the sign); and (2) a uniform, non-steady, incident flow around the traffic sign, whose both intensity and direction are time dependent. The uniform, non-steady flow is the speed generated at the sign position by the moving source representing the vehicle. The load caused by the latter flow on the sign is calculated using two-dimensional strip theory at one section plane on the plate as the simulated vehicles passes.

(4) The pressure field is determined by using the non-steady potential flow Bernoulli equation.

(5) No circulation is produced around the sign, as the vehicle pass is very quick, thus the primary forces on the sign are a result of rapid acceleration of the fluid. Therefore, as shown below, circulatory forces are not developed in the vehicle passage time interval. The characteristic time of vehicle pass is $t_{\mathrm{p}}=L / U_{\infty}(L$ is a characteristic length and $U_{\infty}$ is the vehicle speed). The viscous time scale needed to develop a boundary layer leading to the production of a wake behind the sign, containing vortices, is of the order of $t_{\mathrm{v}}=L^{2} / v$, where $v$ is the kinematic viscosity. Therefore, $t_{\mathrm{p}} / t_{\mathrm{v}}=v /\left(L U_{\infty}\right)=1 / \operatorname{Re}$, where $R e$ is the Reynolds number which usually is $R e \gg 1$. Therefore, the pass is so quick that there is not time enough for circulation to be developed on the flow around the traffic sign.

\section{Vehicle-induced velocity field}

Let us consider the configuration sketched in Fig. 1. The vehicle is represented, in a reference frame $(x, r)$ fixed to the vehicle, by a source placed in an incoming steady, uniform flow of speed $U_{\infty}$ aligned with the $x$-axis (which is the direction of the vehicle motion). The cross-section area of the vehicle is $A_{\mathrm{b}} / 2=\pi r_{t}^{2} / 2$, where $r_{t}$ is the radius of the axisymmetric body. The sign is placed at distance $d=\left|Y_{\mathrm{s}}\right|$ from the $x$ axis. The $x$-axis is the trajectory of the centre position of the moving body. The vehicle itself and its image with regard to the ground plane form the body. The shape of the body obtained in this way is streamlined. An alternative shape, more representative of an actual vehicle could be obtained by using a superposition of 


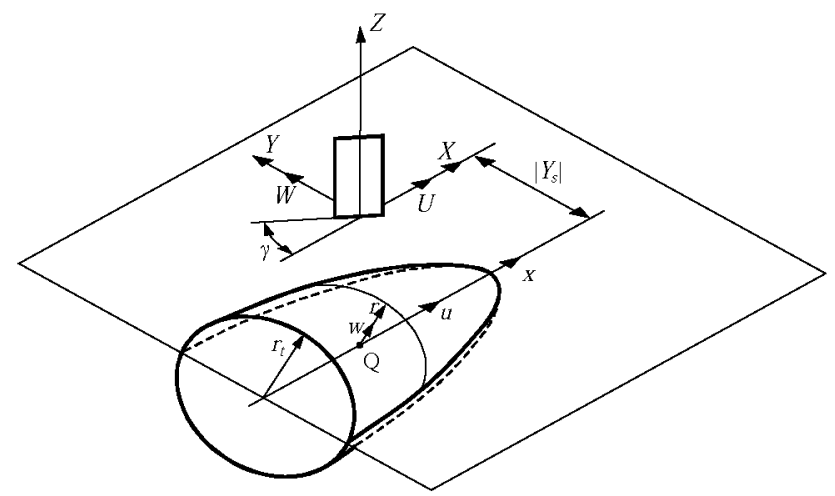

Fig. 1. Model of the flow around a moving vehicle.

sources, sinks and doublets. However, at this stage a rough model of the vehicle was judged to be sufficient.

Using the notation defined in Fig. 1, the velocity field $(u, w)$ in the $(x, r)$ reference frame fixed to the vehicle is given by the superposition of a source of intensity $Q$ and a uniform flow, $U_{\infty}$, as follows:

$$
\begin{aligned}
& u=-U_{\infty}+\frac{Q}{4 \pi} \frac{x}{\left(x^{2}+r^{2}\right)^{3 / 2}}, \\
& w=\frac{Q}{4 \pi} \frac{r}{\left(x^{2}+r^{2}\right)^{3 / 2}} .
\end{aligned}
$$

The source intensity, $Q$, is determined by the evaluation of flux through the body cross-section area far downstream, $A_{\mathrm{b}}$, where the flow speed is close to $U_{\infty}$,

$$
Q=U_{\infty} A_{\mathrm{b}}
$$

and the distance from the vehicle leading edge (stagnation point) to the source position $l_{\mathrm{s}}$ is given by

$$
l_{\mathrm{s}}=\sqrt{\frac{A_{\mathrm{b}}}{4 \pi}}=r_{t} / 2 .
$$

The velocity field $(U, W)$ at a point on the ground plane can be expressed in the ground reference frame $(X, Y, Z)$ by using the coordinate change (with the additional condition that when the vehicle is passing the sign the time is $t=0$, and the position $X=x$ ), as follows:

$$
X=x+U_{\infty} t
$$

and the speed composition

$$
U=u+U_{\infty}
$$


to obtain

$$
\begin{aligned}
& U=\frac{U_{\infty} A_{\mathrm{b}}}{4 \pi} \frac{X-U_{\infty} t}{\left[\left(X-U_{\infty} t\right)^{2}+r^{2}\right]^{3 / 2}}, \\
& W=\frac{U_{\infty} A_{\mathrm{b}}}{4 \pi} \frac{r}{\left[\left(X-U_{\infty} t\right)^{2}+r^{2}\right]^{3 / 2}} .
\end{aligned}
$$

From the point of view of an observer placed at the sign position, the flow impinging on the plate is the velocity field generated by the source at the sign position, $r=-Y_{\mathrm{s}}$, $X=0$ :

$$
\begin{aligned}
& U=-\frac{U_{\infty} A_{\mathrm{b}}}{4 \pi} \frac{U_{\infty} t}{\left[\left(U_{\infty} t\right)^{2}+Y_{\mathrm{s}}^{2}\right]^{3 / 2}}, \\
& W=\frac{U_{\infty} A_{\mathrm{b}}}{4 \pi} \frac{-Y_{\mathrm{s}}}{\left[\left(U_{\infty} t\right)^{2}+Y_{\mathrm{s}}^{2}\right]^{3 / 2}} .
\end{aligned}
$$

The maximum speed experienced by the sign occurs when the vehicle passes at the sign position $(t=0)$ and is given by

$$
\frac{Q}{4 \pi Y_{\mathrm{s}}^{2}}=\frac{U_{\infty}}{4}\left(\frac{r_{t}}{Y_{\mathrm{s}}}\right)^{2}
$$

so that, to give an example, the maximum speed at a distance $Y_{\mathrm{s}}=3 r_{t}$ is $U_{\infty} / 36$, which shows that the vehicle pass do not produces high speeds on the signs, and therefore the load on the sign should be mainly due to the time variation of the velocity potential.

\section{Flow around a flat plate}

The sign (a plate) height is considered to be much larger than the width, so that the flow around it can be considered as two-dimensional (except perhaps close to the sign top). This is not in contradiction with assumption 3 in Section 1 if the sign area is small enough. The sign is standing on the ground.

In order to solve the problem of the flow around the sign, consider the problem sketched in Fig. 2. Let assume that an incoming uniform flow $V_{\infty}$ (yet to be defined) impinges on a plate at an angle $\alpha$. The solution of the two-dimensional problem can be obtained by using the complex potential formulation, $f(\tau)$, where $\tau=X^{\prime}+\mathrm{i} Y^{\prime}$ is the complex variable. As the vehicle passes very quick, it is considered that no circulation is produced around the plate. In this case the problem can be considered as the combination of two problems. The impinging flow can be split in the two components, one along the $X^{\prime}$-axis and the other along the $Y^{\prime}$-axis, i.e. parallel and perpendicular to the plate, respectively. The solution of the first problem, $f_{1}(\tau)$, is trivial, and goes $f_{1}(\tau)=\tau V_{\infty} \cos \alpha$, as the plate does not perturb the incoming flow, which in this problem is parallel to the plate. 


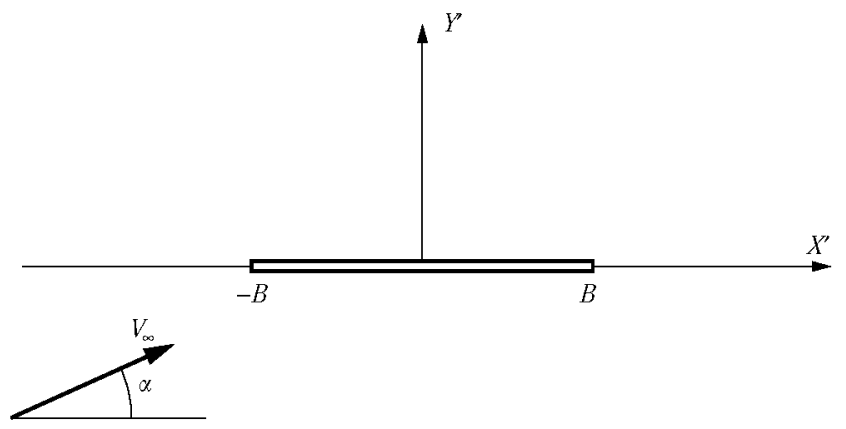

Fig. 2. Model of the flow around the sign parallel to direction of moving vehicle.

The solution of the second problem, a uniform flow (of intensity $V_{\infty} \sin \alpha$, as defined in Eq. (23b)) in the $Y^{\prime}$ direction impinging in a plate, is given by [4]

$$
f_{2}(\tau)=-i V_{\infty} \sin \alpha \sqrt{\tau^{2}-B^{2}},
$$

where $2 B$ is the plate span. From the above expressions the total velocity potential function, $\varphi$, is given by

$$
\varphi\left(X^{\prime}, Y^{\prime}\right)=V_{\infty} \operatorname{Re}\left[\cos \alpha \tau-\sin \alpha \sqrt{\tau^{2}-B^{2}}\right],
$$

where Re denotes real part. In order to determine the total force acting on the plate, the pressure field is needed. It is obtained by using the Bernoulli equation for a nonsteady potential velocity field:

$$
p+\frac{1}{2} \rho|V|^{2}+\rho \varphi_{t}=p_{\mathrm{ref}}+\frac{1}{2} \rho V_{\mathrm{ref}}^{2}+\rho \varphi_{\mathrm{ref} t}
$$

where $p, V$ and $\varphi$ are, respectively, the values of the static pressure, the velocity modulus and the velocity potential function at a point, and $\rho$ is the fluid mass density. The subscript "ref" indicates reference values. The subscript " $t$ " represents partial derivative with respect to time, $\partial / \partial t$. The pressure field is given by

$$
p=-\rho \varphi_{t}-\frac{1}{2} \rho|V|^{2}+g(t)
$$

where

$$
g(t)=p_{\mathrm{ref}}+\frac{1}{2} \rho V_{\mathrm{ref}}^{2}+\rho \varphi_{\mathrm{ref} t}
$$

is a function of the time only, and therefore has no effect on the overall force on the sign. On the plate ( $\tau=X^{\prime}, Y^{\prime}=0$ ) a new (more convenient) variable $\theta$ can be used, defined as follows:

$$
X^{\prime}=B \cos \theta
$$


The velocity and potential function distributions on the plate are given by the expressions:

$$
\begin{aligned}
& \varphi\left(X^{\prime}\right)=V_{\infty}\left[X^{\prime} \cos \alpha \pm \sin \alpha \sqrt{B^{2}-X^{\prime 2}}\right]=V_{\infty}\left[X^{\prime} \cos \alpha \pm B \sin \alpha \sin \theta\right], \\
& |V|=V_{\infty}\left[\cos \alpha \pm \sin \alpha \frac{\left(-X^{\prime}\right)}{\sqrt{B^{2}-X^{\prime 2}}}\right]=V_{\infty}[\cos \alpha \pm \sin \alpha(-\cot \theta)],
\end{aligned}
$$

where the + sign applies to the plate upper surface $\left(Y^{\prime}=0^{+}\right)$and the - sign to the lower surface $\left(Y^{\prime}=0^{-}\right)$. By using Eqs. (13) and (16), the pressure on both the faces of the sign is given by

$$
p=-( \pm) \rho B \sin \theta\left[\dot{V}_{\infty} \sin \alpha+V_{\infty} \dot{\alpha} \cos \alpha\right]-\frac{1}{2} \rho V_{\infty}^{2}[\cos \alpha \pm \sin \alpha(-\cot \theta)]^{2},
$$

where the term $X^{\prime} V_{\infty} \cos \alpha$ has not been included in the contribution of potential because it has the same value on both faces of the plate, and therefore does not contribute to the net force. The dot expresses ordinary derivative with respect to time. The pressure jump across the plate is $\Delta p=p\left(0^{-}\right)-p\left(0^{+}\right)$:

$$
\Delta p=2 \rho B \sin \theta\left[\dot{V}_{\infty} \sin \alpha+V_{\infty} \dot{\alpha} \cos \alpha\right]-\frac{1}{2} \rho V_{\infty}^{2} \sin 2 \alpha \cot \theta .
$$

The first term is symmetric (with regard to the plate middle point) and therefore will contribute to the net force, and not to the net torque around the middle point. The second term is antisymmetric and therefore will not contribute to the force and is responsible for the net torque. Observe that the sign size influences the first term (the one producing the force) but not the torque associated term. The net force, $F$, is perpendicular to the plate and is given by

$$
\begin{aligned}
F & =\int_{-B}^{B} \Delta p \mathrm{~d} X^{\prime}=\int_{0}^{2 \pi} 2 \rho B^{2} \sin ^{2} \theta\left[\dot{V}_{\infty} \sin \alpha+V_{\infty} \dot{\alpha} \cos \alpha\right] \mathrm{d} \theta \\
& =\pi \rho B^{2}\left[\dot{V}_{\infty} \sin \alpha+V_{\infty} \dot{\alpha} \cos \alpha\right] .
\end{aligned}
$$

The net torque, $M_{0}$, with regard to the plate middle point is given by

$$
\begin{aligned}
M_{0} & =\int_{-B}^{B} \Delta p X^{\prime} \mathrm{d} X^{\prime}=-\frac{1}{2} \rho V_{\infty}^{2} B^{2} \sin 2 \alpha \int_{0}^{2 \pi} \cos ^{2} \theta \mathrm{d} \theta \\
& =-\frac{\pi}{4} \rho B^{2} V_{\infty}^{2} \sin 2 \alpha .
\end{aligned}
$$

The net force coefficient, $c_{F}$, and torque coefficient, $c_{M_{0}}$ are defined using the vehicle motion speed, $U_{\infty}$, and the plate width, $2 B$, as follows:

$$
\begin{aligned}
& c_{F}=\frac{F}{\frac{1}{2} \rho U_{\infty}^{2}(2 B)}=\pi B \frac{\dot{V}_{\infty} \sin \alpha+V_{\infty} \dot{\alpha} \cos \alpha}{U_{\infty}^{2}}, \\
& c_{M_{0}}=\frac{M_{0}}{\frac{1}{2} \rho U_{\infty}^{2}(2 B)^{2}}=-\frac{\pi}{4} \frac{V_{\infty}^{2}}{U_{\infty}^{2}} \sin 2 \alpha .
\end{aligned}
$$


To evaluate the effect of the sign on the moving vehicle, the far field due to the plate perturbation should be analysed. From expression (10), in the limit $\tau \rightarrow \infty$ one obtains

$$
f_{2}(\tau) \simeq-\mathrm{i} V_{\infty} \sin \alpha \tau+\mathrm{i} V_{\infty} \sin \alpha \frac{B^{2}}{2 \tau} .
$$

The first term represents the normal component of the impinging flow. The second term represents a doublet, therefore the sign is seen in the far field as a doublet, whose associated velocity field is (in modulus) $V_{\infty} \sin \alpha B^{2} /\left(2|\tau|^{2}\right)$. It means that at a distance 4 times the half-span $B$ the speed experienced due to the sign is $1 / 32$ of the normal component of the speed impinging on the sign, $V_{\infty} \sin \alpha$. This result supports the assumption 3 in Section 1.

\section{Load on the plate}

In order to obtain the load on the plate, the results of Sections 2 and 3 need to be combined. In Fig. 3 the position of plate fixed axes $\left(X^{\prime}, Y^{\prime}\right)$ with regard to ground fixed axes $(X, Y)$ are defined, together with the yaw angle, $\gamma$, the speed orientation angle, $\beta$, and the angle of attack, $\alpha$.

The yaw angle, $\gamma$, is defined by the geometric orientation of the sign with regard to the road direction ( $\gamma=0$ parallel, $\gamma=\pi / 2$ perpendicular to the road direction).

The speed orientation angle, $\beta$, is the instantaneous angle of the speed vector generated by the vehicle motion at the sign position, with the motion direction (axis $x$ or $X$ ):

$$
\tan \beta=\frac{W}{U}=\frac{Y_{\mathrm{s}}}{U_{\infty} t} .
$$

The angle of attack, $\alpha$, is the instantaneous angle of the speed vector with the plate plane,

$$
\alpha=\beta+\gamma .
$$

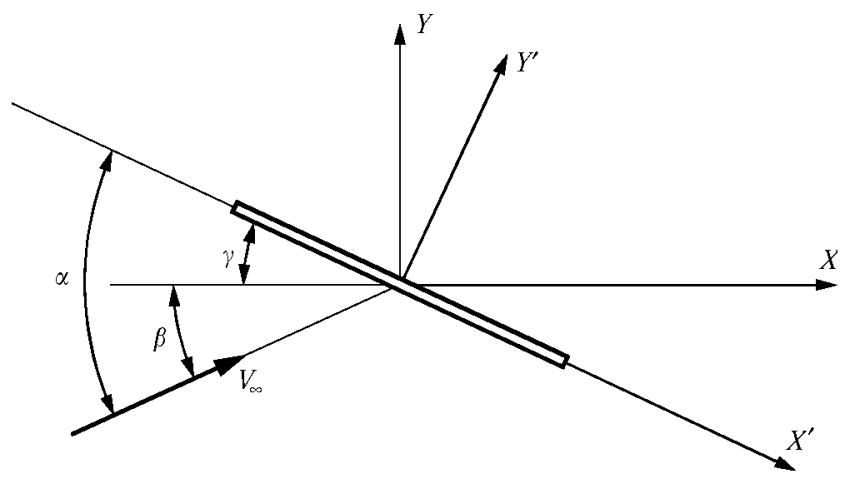

Fig. 3. Model of the flow around the sign at yaw angle $\gamma$. 
The incident speed modulus, $V_{\infty}$, is given by

$$
V_{\infty}=\sqrt{U^{2}+W^{2}}=\frac{U_{\infty} A_{\mathrm{b}}}{4 \pi} \frac{1}{\left(U_{\infty} t\right)^{2}+Y_{\mathrm{s}}^{2}} .
$$

\subsection{Plate parallel to the road}

In this case $\gamma=0$ and therefore, $\alpha=\beta$, and the expression to be substituted in the definition of the force (19) are

$$
\begin{aligned}
& \sin \alpha=\sin \beta=\frac{W}{V_{\infty}}=\frac{-Y_{\mathrm{s}}}{\left[\left(U_{\infty} t\right)^{2}+Y_{\mathrm{s}}^{2}\right]^{1 / 2}}, \\
& \cos \alpha=\cos \beta=\frac{U}{V_{\infty}}=\frac{-U_{\infty} t}{\left[\left(U_{\infty} t\right)^{2}+Y_{\mathrm{s}}^{2}\right]^{1 / 2}} .
\end{aligned}
$$

The force coefficient in the parallel configuration is $c_{F \mathrm{P}}$ :

$$
c_{F \mathrm{P}}=-\frac{3}{4} \frac{B A_{\mathrm{b}} d U_{\infty} t}{\left[\left(U_{\infty} t\right)^{2}+d^{2}\right]^{5 / 2}}=-\frac{3}{4} \frac{B A_{\mathrm{b}}}{d^{3}} \frac{T}{\left[T^{2}+1\right]^{5 / 2}}=-\frac{3}{4} c_{\mathrm{s}} \frac{T}{\left[T^{2}+1\right]^{5 / 2}},
$$

where a new notation for the distance of the sign to the $x$-axis of the vehicle, $d=-Y_{\mathrm{s}}(d>0)$ has been employed to avoid confusion. The configuration parameter, $c_{\mathrm{s}}$, is defined as

$$
c_{\mathrm{s}}=B A_{\mathrm{b}} / d^{3}
$$

and the dimensionless time, $T$ is

$$
T=\frac{t U_{\infty}}{d}
$$

The expression (26) involves two factors, the configuration parameter $c_{\mathrm{s}}$ and the dimensionless time variation. The configuration parameter defined in Eq. (27a) is a dimensionless group that involves the geometrical parameters of the problem. This parameter shows that the force coefficient will increase linearly with both the sign size, $B$, and the vehicle cross section $A_{\mathrm{b}}$, and decrease as the inverse of the third power of the distance $d$.

The force coefficient in parallel orientation, $c_{F \mathrm{P}}$, has been plotted in Fig. 4 as a function of the dimensionless time, $T$. The shape of the curves confirms the experimental results of QBW, which show a pushing of the plate out of the road when the vehicle is approaching $(t<0)$ and a pulling towards the road when the leading edge of the vehicle passes the plate. The extreme values of $c_{F \mathbf{P}}, c_{F \mathrm{Pm}}$, appear at $t=t_{\mathrm{Pm}}\left(\right.$ dimensionless time $\left.T=T_{\mathrm{Pm}}\right)$ :

$$
\begin{aligned}
& t_{\mathrm{Pm}}=\frac{1}{2} \frac{d}{U_{\infty}} ; \quad T_{\mathrm{Pm}}= \pm \frac{1}{2} \\
& \left|c_{F \mathrm{Pm}}\right|=\frac{3}{8}\left(\frac{4}{5}\right)^{5 / 2} \quad c_{\mathrm{s}} \simeq 0.215 \frac{B A_{\mathrm{b}}}{d^{3}} .
\end{aligned}
$$




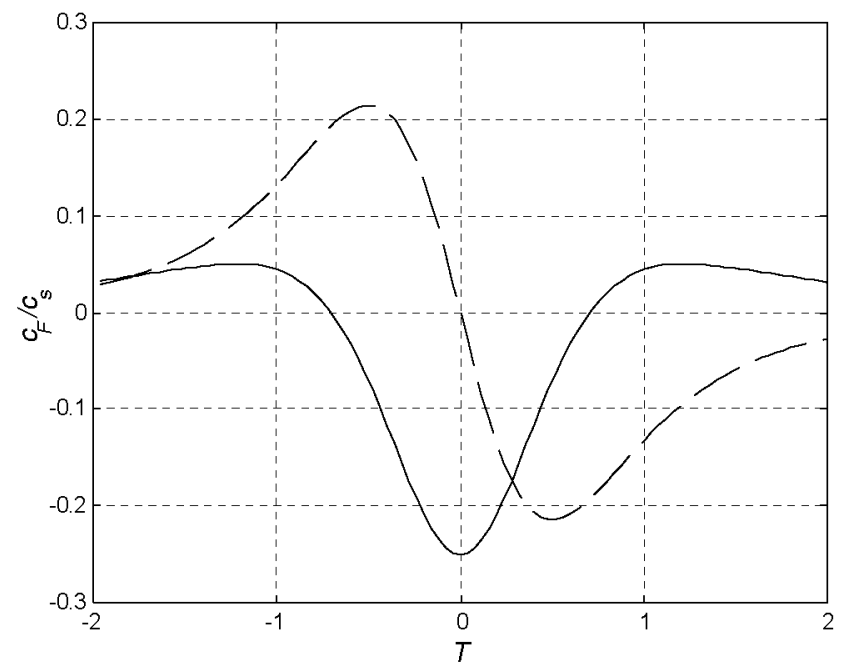

Fig. 4. The force coefficient, $c_{F}$, acting on a sign placed parallel (dashed line) and normal (solid line) to the road.

Observe that the maximum force coefficient increases when the sign size $B$ or the vehicle cross-section area $A_{\mathrm{b}}$ increases, or the distance from the sign to the vehicle, $d$, decreases.

\subsection{Plate normal to the road}

In this case $\gamma=\pi / 2$ and therefore, $\alpha=\beta+\pi / 2$. The force is obtained from Eq. (19):

$$
F=\frac{\rho B^{2} U_{\infty}^{2} A_{\mathrm{b}}}{4 \pi} \frac{2 U_{\infty}^{2} t^{2}-d^{2}}{\left[\left(U_{\infty} t\right)^{2}+d^{2}\right]^{5 / 2}} .
$$

The force coefficient in normal orientation, $c_{F \mathrm{~N}}$, can be expressed as

$$
c_{F \mathrm{~N}}=\frac{1}{4} c_{\mathrm{s}} \frac{2 T^{2}-1}{\left[T^{2}+1\right]^{5 / 2}} .
$$

The variation of $c_{F \mathrm{~N}}$ with dimensionless time, $T$, is also plotted in Fig. 4 . The shape of the curve is quite different than in the parallel case. As the vehicle is approaching the sign $(t<0)$ a small positive peak (in the direction of the vehicle motion) occurs first, but when the vehicle is close (just passing the sign) then a strong negative pulse is produced, pulling the sign in the opposite direction to the vehicle motion.

The time evolution is symmetric with regard to the time origin (the pass of the source in front of the sign). The extreme value of the force coefficient, $c_{F \mathrm{Nm} a}$, occurs at $T=0$ :

$$
\left|c_{F \mathrm{Nm} a}\right|=\frac{1}{4} c_{\mathrm{s}}
$$


and is very similar in magnitude to the force coefficient in the parallel configuration, $c_{F \mathrm{Pm}}$, given by Eq. (29).

Concerning the influence on $c_{F \mathrm{Nm}}$ of the distance from the sign to the vehicle, QBW suggests a variation of $c_{F \mathrm{Pm}}$ as $d^{-2}$ whilst CC propose a $d^{-4}$ law. What is obtained with the present model is something in between, $d^{-3}$. However, in QBW $d$ means the distance from the vehicle side, and here it means distance from the vehicle middle plane. The secondary maxima occur at $t=t_{\mathrm{Nm} b}$ (dimensionless time $\left.T=T_{\mathrm{Nmb}}\right)$ :

$$
T_{\mathrm{Nm} b}= \pm \sqrt{\frac{3}{2}} \simeq \pm 1.22
$$

and the associated relative maximum value is $c_{F \mathrm{Nm} b}$

$$
c_{F \mathrm{Nm} b}=\frac{1}{2}\left(\frac{2}{5}\right)^{5 / 2} c_{\mathrm{s}} \cong 0.051 \frac{B A_{\mathrm{b}}}{d^{3}} .
$$

The ratio of the two extreme absolute values in the parallel configuration is

$$
\frac{c_{F \mathrm{Nm} b}}{\left|c_{F \mathrm{Nm} a}\right|} \simeq 0.20 \text {. }
$$

The ratio of the extreme positions (parallel to normal ratio), relative to the origin is

$$
\frac{T_{\mathrm{Pm}}}{T_{\mathrm{Nm} b}}=0.41 .
$$

\subsection{General case}

If the sign is oriented with regard to the road at a yaw angle, $\gamma$, then, from Eq. (21a) by using Eqs. (22) and (23), the following expression is obtained for the force coefficient, $c_{F}$ :

$$
c_{F}=c_{F \mathrm{~N}} \sin \gamma+c_{F \mathrm{P}} \cos \gamma,
$$

where $c_{F \mathrm{~N}}$ and $c_{F \mathrm{P}}$ are given by Eqs. (31) and (26), respectively. Therefore, in a general case the force on the plate (which is always perpendicular to the plate) is a combination of the results obtained for the parallel and normal configurations. As can be easily checked the force coefficient fulfils the relations:

$$
\begin{aligned}
& c_{F \mathrm{~N}}=c_{F}(\gamma=\pi / 2), \\
& c_{F \mathrm{P}}=c_{F}(\gamma=0) .
\end{aligned}
$$

The variation of $c_{F}$ with dimensionless time, $T$, at different yaw angle values is plotted in Fig. 5. The transition from normal to parallel configuration can be easily appreciated. In the general case the expression for the position of extreme values is not simple because they are given by the solution of a cubic equation. 


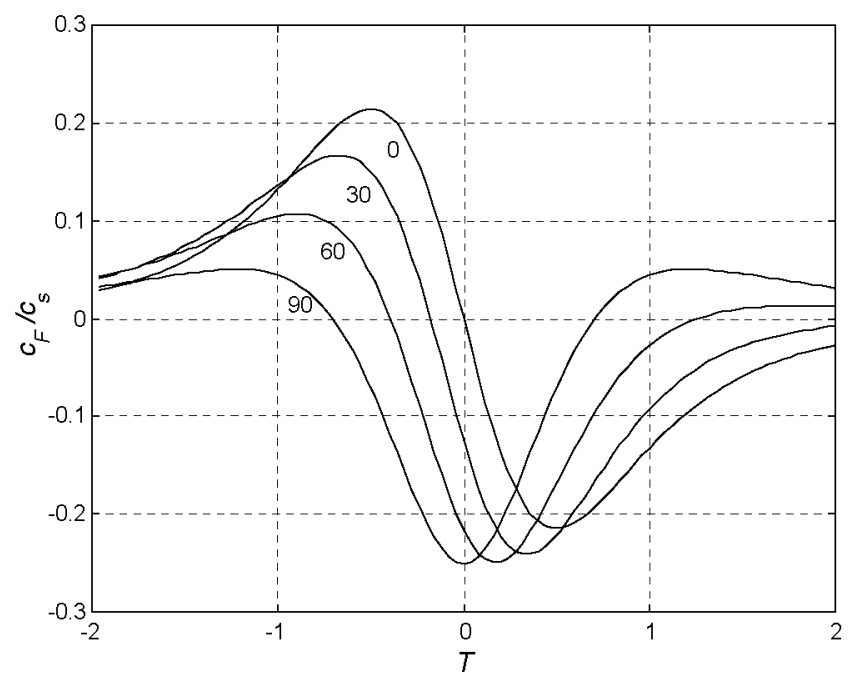

Fig 5. The force coefficient, $c_{F}$, acting on a sign placed at a yaw angle, $\gamma$, with regard to the road. Numbers denote the yaw angle, $\gamma$, in degrees.

\section{Comparison with experimental results}

There are two sources of experimental data available to the authors: QBW and CC. The first of the above-mentioned papers presents data obtained from full scale tests with traffic signs and pedestrians barriers, and the second one presents results obtained from scale model tests performed with overhead highway signs. Both these results are analysed with the help of the theoretical model developed here. The QBW results are considered first.

QBW have tested traffic signs of two different sizes, positioned in two different roads (which means different distances of the sign relative to the road, $d$, different sizes of the sign, $2 B$, and different vehicle motion speed, $U_{\infty}$ ). Comparing QBW's Figs. 3 (750 mm signs), 5 (1500 mm signs) and 8 (pedestrian barriers) with Fig. 5 in the present paper (where the variation of $c_{F}(T)$ is plotted) the close similitude between the experimental and theoretical results can be seen in the case of the normal configuration (Fig. 6). The comparison between theoretical and experimental values of selected parameters is presented in Table 1 . The reference magnitudes considered are the elapsed time between the two first peaks, $t_{\mathrm{Nm} b}$, in the normal configuration, the time elapsed between the time origin and the first peak, $t_{\mathrm{Pm}}$, in the parallel configuration, and the force coefficient, either $c_{F \mathrm{Nm} a}$ or $c_{F \mathrm{Pm}}$, in both the configurations. The values assumed to obtain the theoretical predictions are: for the span, $2 B=750$ and $1500 \mathrm{~mm}$ for the two sign types, and the body cross-section area $A_{\mathrm{b}}=15 \mathrm{~m}^{2}$ (double of the vehicle cross-section).

The theoretical model does not include enough details of the vehicle-sign configuration to produce quantitative agreement between the analytical and experimental results. However, the analytical model captures the experimental 


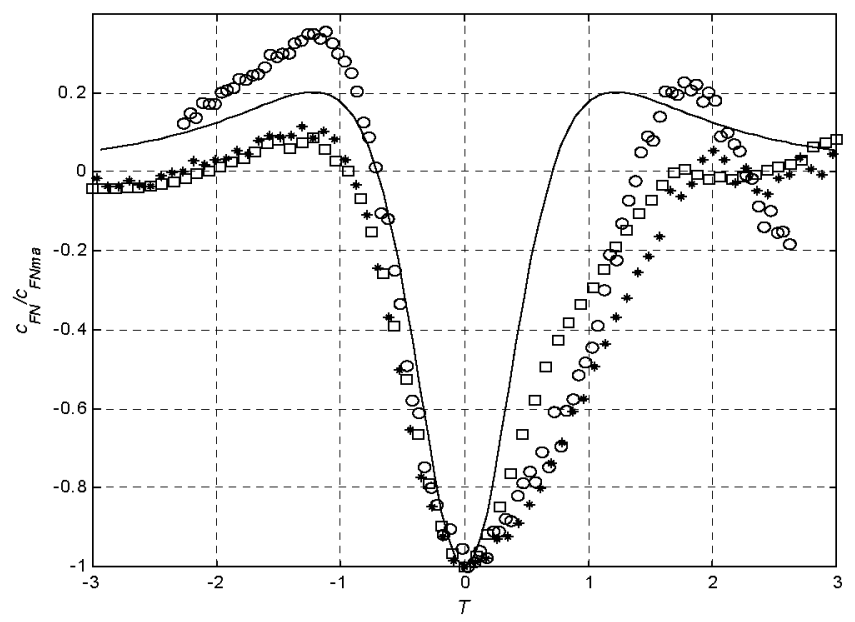

Fig 6. The force coefficient, $c_{F N}$, acting on a sign placed at normal incidence $(\gamma=\pi / 2)$, with regard to the road. $c_{F \mathrm{Nm} a}$, maximum of the absolute value of the force coefficient. Results from: theoretical model (solid line), $750 \mathrm{~mm}$ signs in QBW's Fig. 3 (square), $1500 \mathrm{~mm}$ signs in QBW's Fig. 5 (circle), pedestrian barrier in QBW's Fig. 8 (star).

Table 1

Comparison between experimental and theoretical results

\begin{tabular}{llllllllll}
\hline Ref. $^{(1)}$ & Config $^{(2)}$ & $\operatorname{Road}^{(3)}$ & $U_{\infty}(\mathrm{m} / \mathrm{s})$ & $t_{\mathrm{Nm} b}$ & $t_{\mathrm{Pm}}(\mathrm{s})$ & $d(\mathrm{~m})$ & $t_{\mathrm{Nm} b} * t_{\mathrm{Pm}} *(\mathrm{~s})$ & $c_{F \mathrm{Nm} a} c_{F \mathrm{Pm}}$ & $c_{F \mathrm{Nm} a}{ }^{*} c_{F \mathrm{Pm}}{ }^{*}$ \\
\hline $3^{(\mathrm{a})}$ & $\mathrm{N}$ & $\mathrm{A} 6$ & 27 & 0.12 & $3^{(4)}$ & 0.14 & -0.038 & -0.055 \\
$5^{(\mathrm{a})}$ & $\mathrm{N}$ & $\mathrm{A} 1$ & 31 & 0.24 & $5^{(5)}$ & 0.20 & -0.023 & -0.021 \\
$8^{\text {(b) }}$ & $\mathrm{N}$ & $\mathrm{A} 6$ & 27 & 0.14 & $3^{(4)}$ & 0.14 & -0.023 & -0.070 \\
$8^{(\mathrm{b})}$ & $\mathrm{P}$ & $\mathrm{A} 6$ & 27 & 0.074 & $3^{(4)}$ & 0.056 & 0.015 & 0.059 \\
\hline
\end{tabular}

Experimental data $(\mathrm{QBW}): U_{\infty}$, vehicle speed, $t_{\mathrm{Nm} b}$ and $t_{\mathrm{Pm}}$, elapsed time between two peaks or between a peak and the time origin in normal and parallel configurations, respectively. $d$, distance from the sign middle span to the vehicle middle plane. $c_{F \mathrm{Nm} a}, c_{F \mathrm{Pm}}$, net force coefficient in the normal $(\mathrm{N})$ or parallel $(\mathrm{P})$ configurations, respectively. $\left({ }^{*}\right)$ theoretical results.

(1) The numbers in this column represents the figure number in Ref. QBW.

(2) N: sign normal to the road; P: sign parallel to the road.

(3) UK highway where the tests were performed.

(4) $d=r_{t}+s$, where $r_{t}$ is the equivalent radius of the vehicle $\left(r_{t} \cong 1.5 \mathrm{~m}\right)$ and $s$ is the sign to vehicle separation $(s \cong 1.5 \mathrm{~m})$.

(5) As in Eq. (4) with $r_{t} \cong 1.5 \mathrm{~m}, s=3.5 \mathrm{~m}$ ( $2.5 \mathrm{~m}$ separation plus $1 \mathrm{~m}$ from the centre to the sign side).

(a) Traffic sign. In A6 sign size is $750 \mathrm{~mm}$. In A1 sign size is $1500 \mathrm{~mm}$.

(b) Pedestrian barrier.

behaviour of $c_{F}(T)$ and predicts the correct order of magnitude of the characteristic time and force coefficient.

The largest discrepancies occur in the force coefficient for the pedestrians barriers where the theoretical predictions are up to three times larger than the experimental values, both for the normal and parallel configurations (roughly the same factor for both configurations). 
The results predicted by the theoretical model and experimental data obtained by QBW are shown in Fig. 6. The experimental data are transformed to dimensionless values in the following way. The experimental results $c_{F \mathrm{~N}}$ are rescaled by using the extreme value $c_{F m a}$, the experimental time origin is shifted to the time instant at the extreme value, $t_{\mathrm{m}}$, in order to synchronize the events, and the values of the period between the secondary maxima and the negative peak, $t_{\mathrm{N} b}$ (as shown in Table 1) divided by $\sqrt{3 / 2}$ (see expression (33)) is taken as the characteristic time $t_{\mathrm{c}}$, which should formally be the theoretical characteristic time $t_{\mathrm{c}}=d / U_{\infty}$. Therefore, the conversion to dimensionless time $T$ of the experimental data is obtained by using

$$
T=\frac{t-t_{\mathrm{m}}}{t_{\mathrm{c}}}
$$

Observe the close qualitative agreement between experimental and theoretical results, which is better when $T<0$.

$\mathrm{CC}$ report results for the horizontal loading produced by vehicle-induced gusts on overhead highway sign structures placed normal to the road. In CC work, tests on sign and vehicle models (at 1:30 scale), with the vehicle model passing underneath, have been performed. Several values of test parameters such as the vehicle speed and shape, vehicle length, $l_{\mathrm{v}}$, and height of the sign were considered. In these tests a set of characteristics of the loads were identified, which seems to be universal for all kind of vehicles, e.g. the maximum load appears in the opposite direction to the vehicle motion and occurs when close to the leading edge passing in front of the sign; afterwards the load changes in sign.

Tests on vehicle models with both rectangular parallelepiped shapes and streamlined shape (such as the dividing streamline which appears in the flow generated by a two-dimensional source and an incident uniform flow) were performed by $\mathrm{CC}$, changing the vehicle length, with the traffic sign placed at different heights from the ground level, to study the influence of these parameters.

The main conclusions of $\mathrm{CC}$ with relevance to the present study are summarized as follows:

1. The variation of the force coefficient with time shown in Fig. 7 is a kind of variation which appears in a general situation. The curve $c_{F N}(T)$ presents five peaks (P1-P5) and four zero-crossings (Z1-Z4). The position of $Z 1$ and $P 1$ with regard to peak $\mathrm{P} 2$ (whose position should be considered as the time origin) does not depend on the vehicle length but just on the sign position, as shown in Figs. 7 and 8 of CC, and when the sign height (here denoted as $d$ ) is changed, as shown in CC's Fig. 9, the time coordinate of $\mathrm{Z} 1$ and $\mathrm{P} 1$ with regard to $\mathrm{P} 2$ changes according to the change in $d$, as expressed by Eq. (33).

In fact, the shape of the curve, $c_{F}(T)$ in Fig. 7 could be obtained by superposition of a source $Q_{1}$ placed close to the vehicle leading edge (responsible for peaks P1 and $\mathrm{P} 2$ ), a sink $Q_{2}$ located at a distance of some $0.2 I_{\mathrm{v}}$ (generating peak P3) and another sink (of smaller intensity $Q_{3}$ ) at the vehicle rear (responsible of peak P5). The combination of $Q_{1}$ and $Q_{2}$ is needed to produce a vehicle nose more blunt than the one of a streamlined body obtained with only one source. 


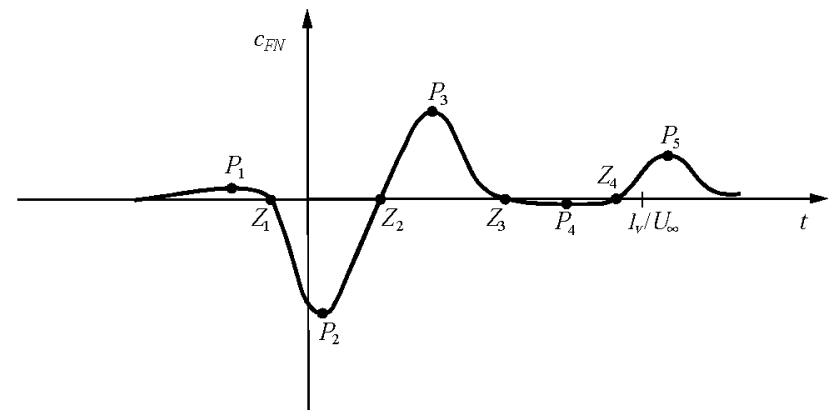

Fig. 7. Typical normal force coefficient, $c_{F \mathrm{~N}}$, variation with time, $t$ (results from $\mathrm{CC}$ ), $U_{\infty}$, vehicle speed. $l_{\mathrm{v}}$, vehicle length, $t$, time.

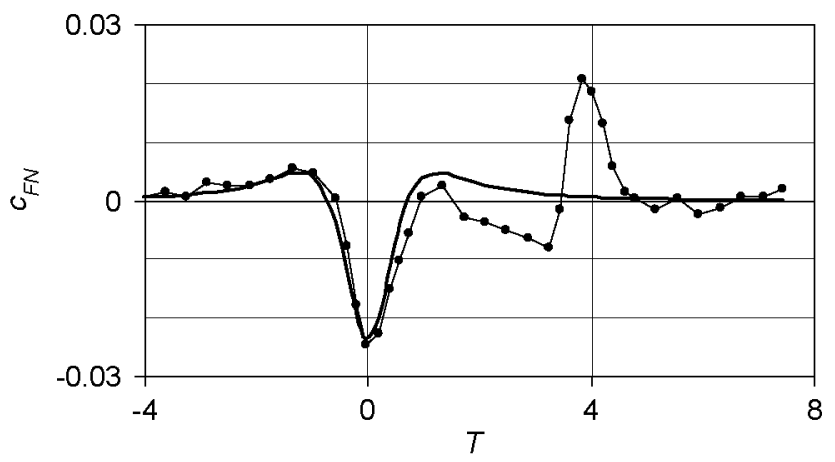

Fig. 8. Comparison of experimental results for normal force coefficient $c_{F N}$ produced by a streamlined body (test $\mathrm{C}$ of $\mathrm{CC}$ ) with theoretical model results (solid line) after reference time offset $(\Delta T=-0.2)$ and length scale matching.

The characteristics of the normal force coefficient $c_{F \mathrm{~N}}(T)$ behaviour close to the leading edge are predicted by the model developed here. In particular, test case $\mathrm{C}$ of $\mathrm{CC}$, which corresponds to a source-shaped body, can be reproduced by the theoretical model with a high fidelity from P1 to P3, Fig. 8, where the test data follow the theoretical pressure profile. Reference time adjustment has been needed in order to match passing time ( $T=0)$ definitions, since during the tests it was measured by means of a photocell at the instant when the vehicle front crossed the sign position. This introduces a time offset for the theoretical model, given by $\Delta T=-\left(l_{\mathrm{s}} / d\right) \approx$ -0.2 , where $l_{\mathrm{s}}$ is the distance from the source location to the actual vehicle front. This time offset and account for the different non-dimensional time scales of the model and experiments was included in processing the test data depicted in Fig. 8. The estimated value of $c_{F \mathrm{Nm} a}$ according to Eq. (32) was $c_{F \mathrm{Nm} a}=0.023$ (taking $A_{\mathrm{b}}=240 \mathrm{~cm}^{2}, B=5.08 \mathrm{~cm}$ and $d=23.5 \mathrm{~cm}$ ) which is very close to the experimental value $c_{F \mathrm{Nm} a}=0.025$.

2. The shape of the vehicle leading edge affects to the intensity of peaks P1-P3. In the case of the streamlined shape (test $\mathrm{C}$ in Fig. 10, of $\mathrm{CC}$ ) the magnitude of these 
peaks is largely reduced compared to the same model vehicle but with box shape. In the streamlined body case, peak P3 is very similar to peak P1 and symmetric to it with regard to P2, just as is predicted in the theoretical model (see Fig. 8).

On the other hand, as shown in the same figure, there is a factor of nearly 10 between peak values at P2 of the force coefficient (for the same model vehicle) from the streamlined shape to box shape, given a feeling of the influence of vehicle front shape influence.

3. The peak P5, which appears close to the trailing edge of the vehicle, is associated by $\mathrm{CC}$ with the effect of a sink placed at the end of the vehicle. As the potential flow that impinges the plate is generated mainly by the changes in the shape of the moving body, this sink would represent the cross-section area changes in the wake when it is formed at the rear of the moving vehicle. As shown in CC's Figs. 9 and 10, the shape of peak P5 seems to be dependent only on the sign height (increases as the sign height decreases) and not on the vehicle length. It is consistent with the fact that the force in this time interval is mostly produced by the vehicle wake, and, according to the present model, it should be almost independent of the vehicle nose shape, and also (for vehicles of the same cross-section shape) only dependent on the sign distance $d$.

\section{Conclusions}

The simple model presented in this paper allows a qualitative explanation of the main characteristics of evolution with time of the force experienced by a traffic sign induced by a vehicle which passes close to it, as reported by several experimenters. In some cases, where the shape of the vehicle is streamlined, a quantitative agreement has been also found.

The time evolution of the force coefficient depends on the orientation of the sign with regard to the traffic motion (antisymmetric for parallel orientation to the road direction and symmetric for normal orientation).

The force coefficient is proportional to a parameter that involves the sign size, the vehicle cross-section area and the distance of the sign to the middle plane of the vehicle.

From the comparison with the available experimental results, it should be pointed out that the force experienced by the sign can be attributed to the transient pressure field due to a potential unsteady flow.

A more detailed model should be developed to extend the applicability of this mathematical model to more complex geometrical configurations. It should incorporate in an appropriate way the boundary conditions due to the vehicle.

\section{Acknowledgements}

The authors would like to thank to Prof. Covert for his help with the access at the data shown in CC, and recognize the contribution of the European Programme COST 14. The authors are also indebted with Profs. J. Meseguer and J.M. Perales 
for helpful discussions. Furthermore, they want also to thank the referees for their constructive comments.

\section{References}

A.D. Quinn, C.J. Baker, N.G. Wright, Wind and vehicle induced forces on flat plates. Part 2: vehicle induced force, J. Wind Eng. Ind. Aerodyn. 89 (2001) 831-847.

P.M. Cali, E.E. Covert, Experimental measurements of the loads induced on an overhead highway sign structure by vehicle-induced gusts, J. Wind Eng. Ind. Aerodyn. 84 (2000) 87-100.

A. Sanz-Andrés, J. Santiago-Prowald, Train-induced pressure on pedestrians, J. Wind Eng. Ind. Aerodyn. 90 (2002) 1007-1015.

H. Ashley, M. Landahl, Aerodynamics of wings and bodies, 1985, Dover, New York, p. 112. 